\title{
Duration of hexobarbital-induced sleep and monoamine oxidase activities in rat brain: Focus on the behavioral activity and on the free-radical oxidation
}

\author{
Vadim E. Tseilikman ${ }^{1}$, Denis A. Kozochkin ${ }^{1}$, Eugenia B. Manukhina ${ }^{2,3}$, H. Fred Downey ${ }^{3}$, Olga B. \\ Tseilikman $^{4}$, Maria E. Misharina ${ }^{1}$, Anna A. Nikitina ${ }^{1}$, Maria V. Komelkova ${ }^{1}$, Maxim S. Lapshin ${ }^{4}$, \\ Marina V. Kondashevskaya ${ }^{5}$, Svetlana S. Lazuko ${ }^{6}$, Oxana V. Kusina ${ }^{1}$ and Marat N. Sahabutdinov ${ }^{1}$ \\ ${ }^{1}$ South-Ural State Medical University, Chelyabinsk, Russia \\ ${ }^{2}$ University of North Texas Health Science Center, Fort Worth, Texas, USA \\ ${ }^{3}$ Institute of General Pathology and Pathophysiology, Moscow, Russia \\ ${ }^{4}$ South-Ural State University, Chelyabinsk, Russia \\ ${ }^{5}$ Federal State Budgetary Scientific Institution "Scientific Research Institute of Human Morphology", Moscow, Russia \\ ${ }^{6}$ Vitebsk State Medical University, Vitebsk, Belarus
}

\begin{abstract}
The present study is focused on the relationship between monoamine oxidase (MAO) activity and hepatic content of cytochrome P450 (CYP), which reflects the status of microsomal oxidation. For vital integrative evaluation of hepatic microsomal oxidation in rats, the hexobarbital sleep test was used, and content of CYP was measured in hepatic microsomes. Rats with short hexobarbital sleep time (SHST) had higher content of microsomal CYP than rats with long hexobarbital sleep time (LHST). Whole brain MAO-A and MAO-B activities, serotonin and carbonylated protein levels were higher in SHST than in LHST rats. MAO-A and MAO-B activities were higher in brain cortex of SHST rats; MAO-A activity was higher only in hypothalamus and medulla of LHST. The same brain regions of LHST rats had higher concentrations of carbonylated proteins and lipid peroxidation products than in SHST rats. MAO activity was correlated with microsomal oxidation phenotype. Rats with higher hepatic content of CYP had higher activities of MAO-A and MAO-B in the brain and higher plasma serotonin levels than rats with lower microsomal oxidation. In conclusion, data obtained in this study showed a correlation between MAO activity and microsomal oxidation phenotype.
\end{abstract}

Key words: Cytochrome P450 (CYP) - Hexobarbital-induced sleep - Open field locomotion Oxidative stress - Serotonin

Abbreviations: CYP, cytochrome P450; HST, hexobarbital-induced sleep time; LHST, long HST duration; MAO, monoamine oxidase; SHST, short HST duration.

\section{Introduction}

Monoamine oxidase (MAO) is an enzyme bound to the outer membrane of mitochondria and it plays a key role in intracellular metabolism of phenyl- and indola-

Correspondence to: Vadim E. Tseilikman, Biochemistry Department South Ural State Medical University, Vorovskogo 64, Chelyabinsk 454092, Russia

E-mail: vadimed@yandex.ru lkylamines (Nagatsu 2004). MAO consists of two isoforms, MAO-A and MAO-B, which are differ in their sensitivity to the acetylene inhibitors, chlorgiline, pargyline and deprenyl, substrate specificity, and dependence on the lipid microenvironment (Chen and Shih 1998). MAO-A preferentially deaminates norepinephrine and serotonin (Nagatsu 2004) and loses its activity upon depletion of mitochondrial phospholipids (Ekstedt and Oreland 1976). $\mathrm{O}$-phenylethylamine and dopamine are specific substrates for MAO-B (Glover et al. 1977; Abel and Kwan 2001). 
The major physiological function of MAO is metabolism of monoamine neurotransmitters. Changes in the rate of biogenic amine deamination in nerve tissue play a significant role in the pathogenesis of mental disorders (Brunner et al. 1993; Furlong et al. 1999; Duncan et al.2012). The severity of depressive disorder correlates with increased MAO activity and decreased brain levels of serotonin and norepinephrine (Tipton et al. 1992; Miura et al. 1993). Cytochrome P450 (CYP) is a hepatic enzyme, which contributes to the metabolization of MAO inhibitors; these MAO inhibitors are used for the treatment of depression and Parkinson disease (Spina 2012). Recently, the role of monoamine neurotransmitters in the regulation of the liver CYP activity was demonstrated (Kot et al. 2015; Rysz et al. 2015). MAO, as a key factor in the monoamine metabolism, could therefore be involved in the regulation of the CYP activity. However, the relationship between MAO activity and the intensity of microsomal oxidation has not been sufficiently studied. Hexobarbital-induced sleep time (HST) is considered as a tool to assess hepatic microsomal oxidation (Papas et al. 1993; Cherala et al. 2007). Since monoamines play an important role in the regulation of hepatic microsomal oxidation, it is possible that the duration of phenobarbital-induced sleep is depending on the metabolism of biogenic amines. It will be therefore useful to use HST as an indicator for the intensity of MAO-dependent processes in the brain. Since MAO is involved in the regulation of anxiety and in metabolism of biogenic amines, the cerebral levels of MAO-A, MAO-B and serotonin and the duration of open-field locomotion in the rats with different duration of hexobarbital-induced sleep time were compared. In addition, taking into consideration the importance of MAO in the regulation of oxidative stress, we compared MAO activity in brain structures with concentrations of lipid peroxidation products and carbonylated proteins in rats with different HST duration of hexobarbital-induced sleep time. This pilot was focused on a correlation between hepatic microsomal oxidation in liver and MAO-dependent processes in brain.

\section{Materials and Methods}

\section{Animals}

Sixty male Sprague-Dawley rats were used in this study. Rats were housed in standard rat cages and fed rat chow and tap water ad libitum. The temperature in the housing facility was controlled at $21^{\circ} \mathrm{C}$, and lights were set to a 12:12-h light-dark cycle, with lights on at 7:00 a.m. Rats were acquired from the breeding facility "Pushchino" (Pushchino, Russia) and allowed three weeks to acclimate before the initiation of the study protocol; during this time they were handled and weighed once per day (7 times per week) to accustom them to this procedure. The investigation conforms to the "Guide for the Care and Use of Laboratory Animals" published by the National Institutes of Health (NIH publication 85-23, revised in 2011), and the study was approved by the South Ural State Medical University Ethics Review Board.

\section{Hexobarbital-induced sleep}

Duration of the sleep induced by the barbiturate derivative, hexobarbital, was used to assess the activity of hepatic microsomal oxidation (Lewis et al. 1997). Depending on the HST, animals are characterized as fast and slow metabolizers.

Hexobarbital solution (prepared freshly on the day of the experiment) was administered as a $60 \mathrm{mg} / \mathrm{kg}$ i.p. dose. HST was designated as the time between injection and recovery of the righting reflex, which was defined as the ability of the animal, when placed on its back on a flat surface, to turn over on its paws three times in $15 \mathrm{~s}$. Based on results of the HST test, rats were divided into two groups: rats with short HST duration (SHST, <15 min) and long HST duration (LHST, >15 min).

\section{Behavioral activity}

Behavioral activity of rats was tested 14 days after the HST test using the open field apparatus. The apparatus consisted of a circular area $(110 \mathrm{~cm}$, i.d.) with $65 \mathrm{~cm}$ high walls. The floor was divided into a grid of 12 peripheral and 4 central segments. The following behavioral parameters were recorded visually: 1) horizontal locomotor activity, 2) number of vertical movements (rearings), 3) grooming, and 4) defecation (bolus number), which reflects the autonomic reaction of fear. Open field behavior was evaluated by an observer blinded to the group assignment. The test was started by placing a rat in one of the peripheral segments with the head directed to the wall. Each test lasted for $10 \mathrm{~min}$.

\section{Sample collection and treatment}

On the $15^{\text {th }}$ day after the HST test, rats were sacrificed with diethyl inhalation anesthesia $(2.75 \mathrm{ml} / \mathrm{l}$ air $)$. For measuring plasma serotonin concentration, blood was collected from the retro-orbital sinus into heparinized tubes and centrifuged at $1000 \times g$ for $10 \mathrm{~min}$ in a refrigerated centrifuge. Collected plasma was stored at $-80^{\circ} \mathrm{C}$.

For measuring cytochrome P450 content in hepatic microsomes, the liver was isolated and washed in cold $0.9 \%$ $\mathrm{NaCl} .500 \mathrm{mg}$ samples were prepared and stored in liquid nitrogen. 
For measuring MAO-A and MAO-B activities, carbonylated proteins and lipid peroxidation products, brain structures were isolated as described by Glovinski and Iversen (1966). The following brain regions were isolated: cortex, hypothalamus, midbrain, hippocampus, cerebellum, and medulla with pons. The whole brain was used for measuring MAO-A and MAO-B, carbonylated proteins, lipid peroxidation products, and serotonin. Changes observed in the brain structures were compared to changes in the whole brain.

\section{Measurement of total CYP content in hepatic microsomes}

Livers were homogenized in $1.15 \% \mathrm{KCl}$. The homogenates were centrifuged at $9,000 \times g$ for $20 \mathrm{~min}$, and the supernatant was then centrifuged at $100,000 \times g$ for $60 \mathrm{~min}$. Microsomal pellets were resuspended in $0.1 \mathrm{M}$ Tris- $\mathrm{HCl}$ buffer $(\mathrm{pH} 7.4)$ containing $0.5 \mathrm{mM}$ dithiothreitol, $0.1 \mathrm{mM}$ EDTA, and 20\% glycerol. Microsomal protein concentrations were determined by the Bradford protein assay method, using the Bio-Rad Protein Assay kit (Bio-Rad, Hercules, CA, USA) and bovine serum albumin (BSA; Sigma-Aldrich Inc., St. Louis, MI, USA) as the standard, according to the protocol provided by the manufacturer. The total CYP content were quantified from carbon monoxide difference spectrum of the dithionite-reduced proteins between $450 \mathrm{~nm}$ and $490 \mathrm{~nm}$, using an extinction coefficient $91 \mathrm{mM} \mathrm{m}^{-1} \cdot \mathrm{cm}^{-1}$ as described by Omura and Sato (1964).

\section{Measurement of MAO activities in brain tissue}

Brain activities of MAO-A and MAO-B in rats' mitochondria were measured as described by Tipton et al. (2006). Brain homogenate was prepared in $0.067 \mathrm{M}$ sodium phosphate buffer (e.g., 1/10 w/v; pH = 7.2) and centrifuges with isolation of mitochondria. The isolation of brain mitochondria was carried out according to the method reported by Satav and Katyare (2004).

Before adding specific substrates, mitochondria were preincubated with a selective, irreversible inhibitor of one of the MAO isoforms for $60 \mathrm{~min}$ at $37^{\circ} \mathrm{C}$. Thus, MAO-A activity was measured in the presence of selegeline, a selective MAO-B inhibitor, and MAO-B activity was measured in the presence of clorgeline, a selective MAO-A inhibitor. For inhibition of MAO-A or MAO-B activity, $100 \mu \mathrm{l}$ of 1 $\mu \mathrm{M}$ clorgeline or $100 \mu \mathrm{l}$ of $0.5 \mu \mathrm{M}$ selegeline, respectively, was added to $1 \mathrm{ml}$ of mitochondrial suspension containing $\mathrm{MAO}$ in the membrane-bound form and incubated for $60 \mathrm{~min}$ at $37^{\circ} \mathrm{C}$. For measuring the MAO-A activity, $4 \mathrm{mM}$ 5-hydroxytriptamine creatinine sulfate was used as a substrate. For measuring the MAO-B activity $13.32 \mathrm{mM}$ bensylamine hydrochloride was used as a substrate. Aldehyde content was measured at $250 \mathrm{~nm}$ and $278 \mathrm{~nm}$ for MAO-A and MAO-B, respectively.

\section{Measurement of lipid peroxidation products}

$0.5 \mathrm{ml}$ of brain tissue homogenate suspended in $0.9 \%$ $\mathrm{NaCl}$ containing $0.1 \%$ EDTA was extracted with heptane-isopropanol mixture $(1: 1, \mathrm{v} / \mathrm{v})$ (Volchegorskii et al. 1989). This method allows differentiating acyl peroxides from phospholipids which are extracted mostly into the isopropanol phase, and non-esterified intermediates of fatty acid peroxides, which are extracted primarily into the heptane-phase. The extract was cleaned from the protein precipitate and other corpuscular impurities by centrifugation. The clarified extract was diluted with $5 \mathrm{ml}$ of heptane-isopropanol mixture (3:7, v/v). Two $\mathrm{ml}$ of hydrochloric acid $(\mathrm{pH}=2)$ were added to the diluted lipid extract for 30 minutes for complete phase separation. The upper (heptane) phase was carefully decanted and transferred to a separate tube. Results were expressed as the oxidation index, for ketodienes and conjugated trienes $(\mathrm{KD}+\mathrm{CT})$.

\section{Measurement of protein oxidation products}

Brain protein carbonyls were measured using the method described by Levine et al. (1990) which involves using 2,4dinitrophenylhydrazine as a reagent and estimating the 2,4-dinitrophenylhydrazone derivative content in proteins. The carbonyl content was measured at $430 \mathrm{~nm}$ and expressed as nmol protein carbonyls per mg of tissue. Protein concentration was determined according to the Bradford method using the Bio-Rad Protein Assay kit (Bio-Rad, Hercules, CA, USA).

\section{Measurement of serotonin}

In plasma as well as in brain, serotonin was assayed using an ELISA kit (Biosource, Nivelle, Belgium) following the procedure recommended by the manufacturer. For this assay, the lowest level of sensitivity is $0.5 \mathrm{ng} / \mathrm{ml}$ (Papaliodis et al. 2008).

\section{Statistical analyses}

The non-parametric Mann-Whitney $U$-test was used to determine significance of intergroup difference for groups with different duration of hexobarbital-induced sleep. This test was used due to the absence of normal distribution and the presence of only two groups of animals. The statistical treatment was performed using the program package Statistica for Windows, ver.8. All measurements were done in duplicate or triplicate, and mean values were computed for each rat. Values are presented as means \pm SD. Associations between measured parameters were examined using Spearman's correlation analysis. 


\section{Results}

Correlation between duration of hexobarbital-induced sleep and liver content of CYP

HST is considered an integral index of hepatic microsomal oxidation. Figure 1 shows that $47 \%$ of rats had shorter HST $(11.3 \pm 1.8 \mathrm{~min})$ and $53 \%$ had long HST $(39.1 \pm 11.2 \mathrm{~min}$, $p=0.006$ ). CYP content (Fig. 2) in hepatic microsomes was higher $(1601 \pm 403 \mathrm{ng} / \mathrm{mg}$ protein) than in the LHST group $(1035 \pm 320 \mathrm{ng} / \mathrm{mg}$ protein; $p=0.044)$. HST negatively correlated with microsomal CYP level ( $\mathrm{Rs}=-0.89 ; p=0.006)$.

\section{Open field test}

CYP isoforms contribute to regulation of glucocorticoids, androgens, estrogens and other endocrine and metabolic factors involved in regulation of behavior.

In the open field test (Fig. 3A), frequency of anxiogenic defecation was higher in rats with SHST (5.3 \pm 1.1 boluses $/ 10 \mathrm{~min})$ than in rats with LHST $(1.4 \pm 0.5$ boluses/10 $\mathrm{min}, p=0.021$ ). In addition (Fig. 3B), SHST rats had lower horizontal locomotor activity $(39.1 \pm 9.4$ crossed sectors/10 $\mathrm{min})$ compared with LHST rats $(71.5 \pm 18.7$ crossed sectors $/ 10 \mathrm{~min}, p=0.019)$. These groups were not statistically different in orientation activity determined by the number of rears and grooming acts.

HST and horizontal locomotor activity were positively correlated ( $\mathrm{Rs}=0.635 ; p=0.024)$. Also, the frequency of anxiogenic defecation positively correlated with microsomal level of CYP (Rs $=0.615 ; p=0.037)$ and negatively correlated with HST $(\mathrm{Rs}=-0.58 ; p=0.046)$. These correlations indicate a relationship between microsomal oxidation and

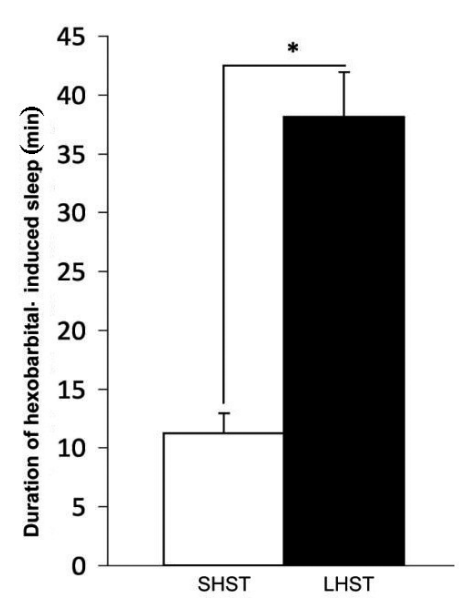

Figure 1. Duration of hexobarbital-induced sleep. Empty bar, SHST group; black bar, LHST group. ${ }^{*}$ Significant difference between groups, $p<0.01$. behavioral parameters. We suggest that these correlations reflect biochemical regulation of transmitters involved in behavioral reactions, including biogenic amines, such as noradrenalin, serotonin and dopamine which are known to be metabolized by MAO.

\section{Correlations between brain MAO activity and hepatic CYP in rats with different HST}

Figures 3 show that in SHST rats, which had greater anxiogenic defecation and lesser locomotor activity than LHST rats, whole brain MAO-A and MAO-B activities were higher by $50 \%(p=0.024)$ and $84 \%(p=0.044)$, respectively, than in the LHST group. Duration of hexobarbital-induced sleep negatively correlated with activities of both $\mathrm{MAO}-\mathrm{A}$ (Rs = $-0.721 ; p=0.024)$ and MAO-B (Rs $=-0.613 ; p=0.029)$. In addition, brain MAO-A activity positively correlated with hepatic content of CYP (Rs $=0.633 ; p=0.027)$.

\section{MAO-A and MAO-B activities in brain structures of rats with different HST}

Changes in MAO-A and MAO-B (Fig. 4) activities in the cerebral cortex were consistent with changes in MAO-A and MAO-B in the whole brain. Specifically, SHST rats had higher cortical MAO-A activity $(0.327 \pm 0.048 \mathrm{nmol} / \mathrm{min} /$ $\mathrm{mg}$ protein $v s .0 .187 \pm 0.039 \mathrm{nmol} / \mathrm{min} / \mathrm{mg}$ protein, respectively, $p=0.037)$ and MAO-B activity $(1.564 \pm 0.432 \mathrm{nmol} /$ $\mathrm{min} / \mathrm{mg}$ protein $v s .0 .823 \pm 0.241 \mathrm{nmol} / \mathrm{min} / \mathrm{mg}$ protein, respectively, $p=0.044$ ) than LSHT rats. As in the whole brain, cortical MAO-A activity negatively correlated with HST $(\mathrm{Rs}=-0.707 ; p=0.028)$ and positively correlated with hepatic level of CYP $(\mathrm{Rs}=0.697 ; p=0.023)$.

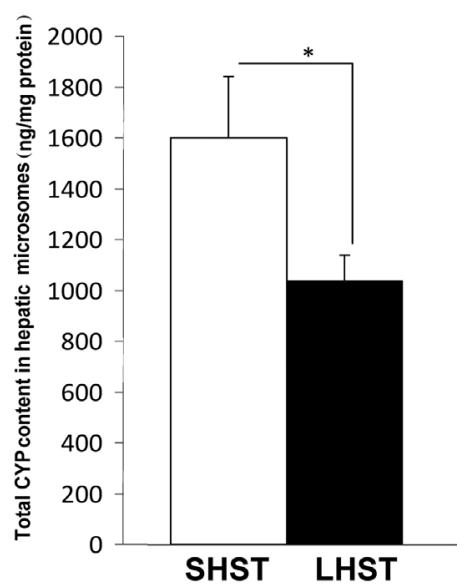

Figure 2. Cytochrome P450 (CYP) concentration in hepatic microsomes. SHST rats (empty bar) had higher CYP concentration than LHST rats (black bar). ${ }^{*}$ Significant difference between groups, $p<0.05$. 
In the hypothalamus, midbrain and medulla (Fig. 4) of SHST rats, MAO-A activity was significantly higher than in LHST rats (59\%, 66\%, and 240\%, respectively). Activities of MAO-B were also slightly higher in hypothalamus and midbrain of SHST than LHST rats, although these differences were not statistically significant. However in the medulla, consistent with the MAO-A activity, MAO-B activity was $220 \%$ higher in SHST than in LHST rats $(p=$ 0.027).

HST negatively correlated with MAO-A activity in the cortex $(\mathrm{Rs}=-0.617 ; p=0.023)$, midbrain (Rs $=-0.695 ; p=$ 0.014 ), and medulla (Rs $=-0.578 ; p=0.044)$. No statistically significant differences were found between hippocampal MAO-A and MAO-B activities in the SHST and LHST groups.

\section{Brain and blood serotonin levels in rats with different HST}

In spite of different MAO-A activities in brain of SHST and LHST rats, statistically significant differences in brain serotonin levels were absent $(813.58 \pm 467.447 \mathrm{ng} / \mathrm{ml} v s .768 .25$ $\pm 250.59 \mathrm{ng} / \mathrm{ml}$, respectively, $p=0.16$ ) (Fig. $5 \mathrm{~A}$ ). However,
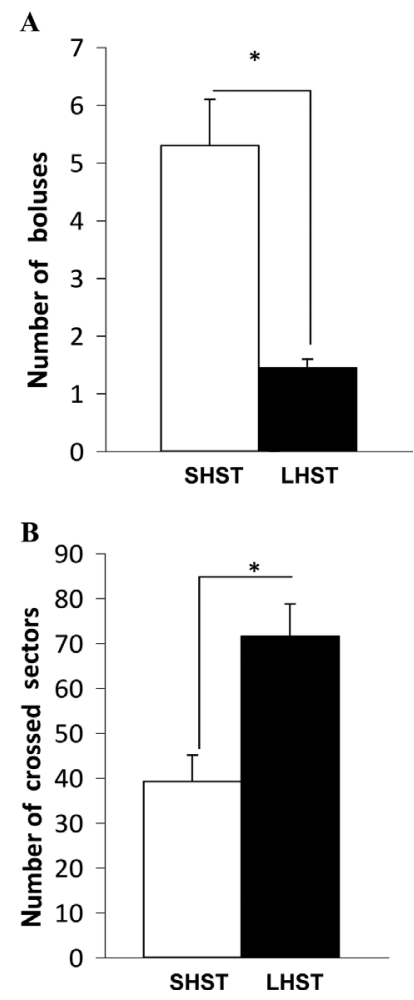

Figure 3. Results of open field test in SHST (empty bar) and LSHT (black bar) rats. A. Anxiogenic defecation. Number of boluses for 10 min was greater in SHST than in LHST rats. B. Horizontal locomotor activity. Number of crossed sectors for $10 \mathrm{~min}$ was less in SHST than in LHST rats. ${ }^{\star}$ Significant difference between groups, $p<0.05$. plasma serotonin was higher in SHST than in LHST rats $(197.24 \pm 45.33 \mathrm{ng} / \mathrm{ml} v s .138 .76 \pm 15.29 \mathrm{ng} / \mathrm{ml}$, respectively, $p=0.025)$ (Fig. 5B). Thus, in rats with SHST and LHST, the difference in MAO activities was not consistent with difference in brain serotonin level. Likewise, the difference in behavioral activity was not consistent with the difference in brain serotonin level. Therefore, MAO-induced behavioral differences are not related directly with changes in serotonin level. However, these behavioral differences may be related with the MAO-mediated increase in oxidative stress.

\section{Oxidative stress in brain}

MAO activation is associated with $\mathrm{H}_{2} \mathrm{O}_{2}$, which can initiate free-radical oxidation of proteins and lipids. Figure 6 shows
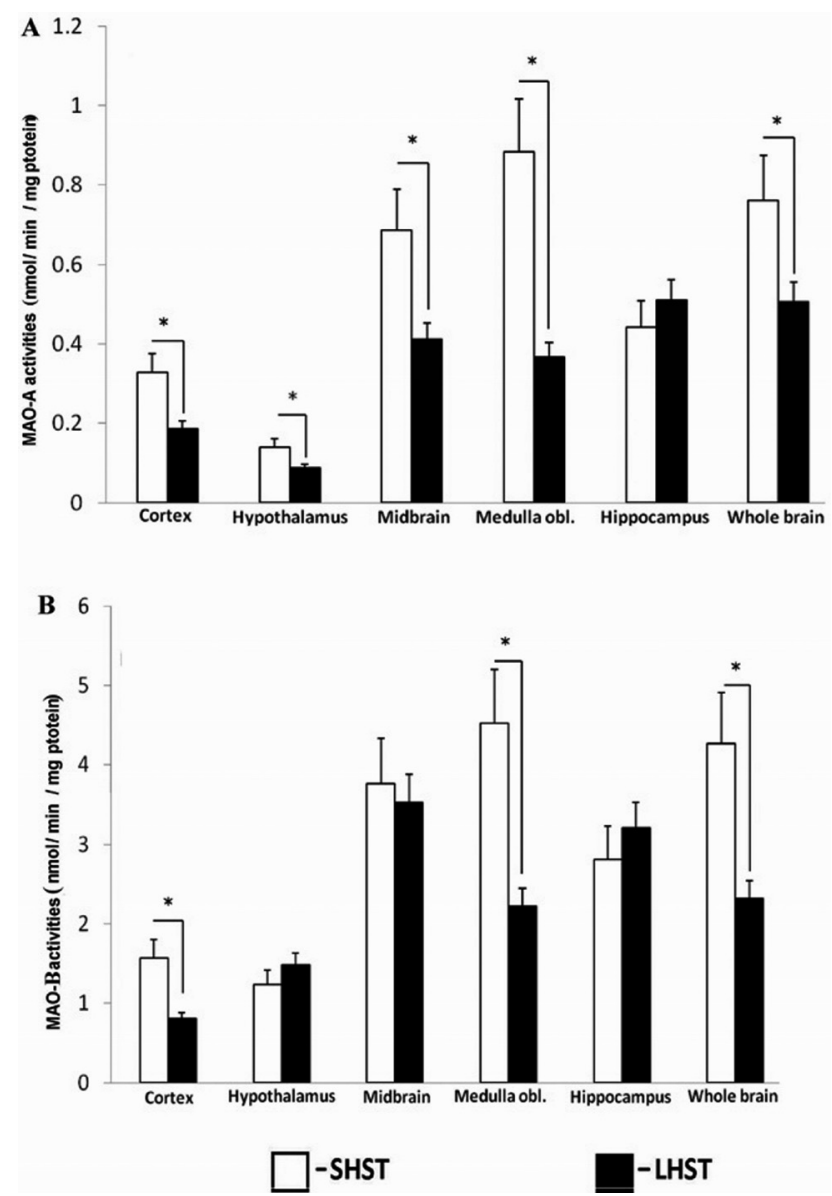

Figure 4. A. MAO-A activities in rat brain. In the whole brain, cortex, midbrain and medulla, MAO-A activities were higher for the SHST group (empty bars) than the LHST group (black bars). B. MAO-B activities in rat brain regions. In the whole brain, cortex and midbrain, MAO-B activities were higher for the SHST group than the LHST group. * Significant difference between groups, $p<0.05$. 
that the content of carbonylated proteins was higher in the whole brain of SHST rats than in LHST rats $(p=0.043)$. In addition, in the SHST group compared to the LHST group, the content of carbonylated proteins was higher in the brain cortex $(p=0.014)$ and the hypothalamus $(p=0.032)$.

In the hypothalamus of SHST rats, in addition to higher carbonylated proteins, heptane-soluble ketodiens and conjugated triens were also higher than in LHST rats (Fig. 7) $(p=0.011)$. Higher concentrations of these lipid peroxidation products were also observed in the cortex, hippocampus and medulla as well as in the whole brain (Fig. 7).

The MAO-A activity positively correlated with concentrations of heptane-soluble ketodiens and conjugated triens in the brain ( $\mathrm{Rs}=0.62 ; p=0.021$ ); the MAO-B activity positively correlated with the content of carbonylated proteins ( $\mathrm{Rs}=0.57 ; p=0.034)$. These correlations suggest a causative relationship between MAO activity and free-radical oxidation in the brain.

Positive correlations observed in the midbrain between the MAO-A activity and the content of carbonylated proteins ( $\mathrm{Rs}=0.627 ; p=0.021)$ show a role of MAO in upregulation of free-radical oxidation.

\section{A}

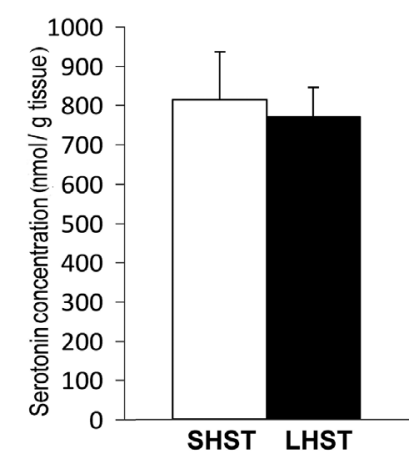

B

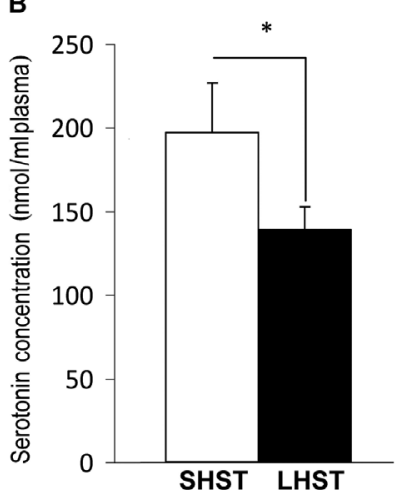

Figure 5. A. Serotonin concentration in whole brain. Brain serotonin concentration did not significantly differ between SHST (empty bar) and LHST groups (black bar). B. Serotonin concentration in blood plasma. Serotonin level was higher in SHST rats than in LHST rats. ${ }^{\star}$ Significant difference between groups, $p<0.05$.
The positive correlation found in the whole brain between the content of carbonylated proteins and anxiogenic defecation $(\mathrm{Rs}=0.729 ; p=0.017)$ indicates a relationship between behavioral reactions and free-radical oxidation. A negative correlation was observed between the cortical level of carbonylated proteins and the locomotor activity ( $\mathrm{Rs}=0.623 ; p=$ 0.025). The locomotor activity also negatively correlated with

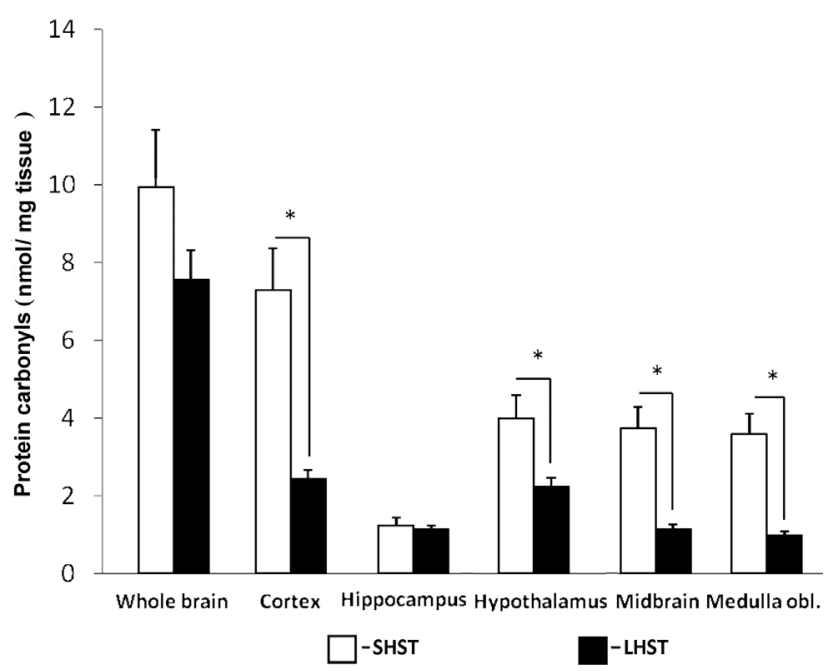

Figure 6. Concentration of carbonylated proteins in rat brain. In cortex, hypotalamus, midbrain and medulla, concentrations of carbonylated proteins were higher for SHST rats (empty bars) than for LHST rats (black bars). ${ }^{*}$ Significant difference between groups, $p<0.05$.

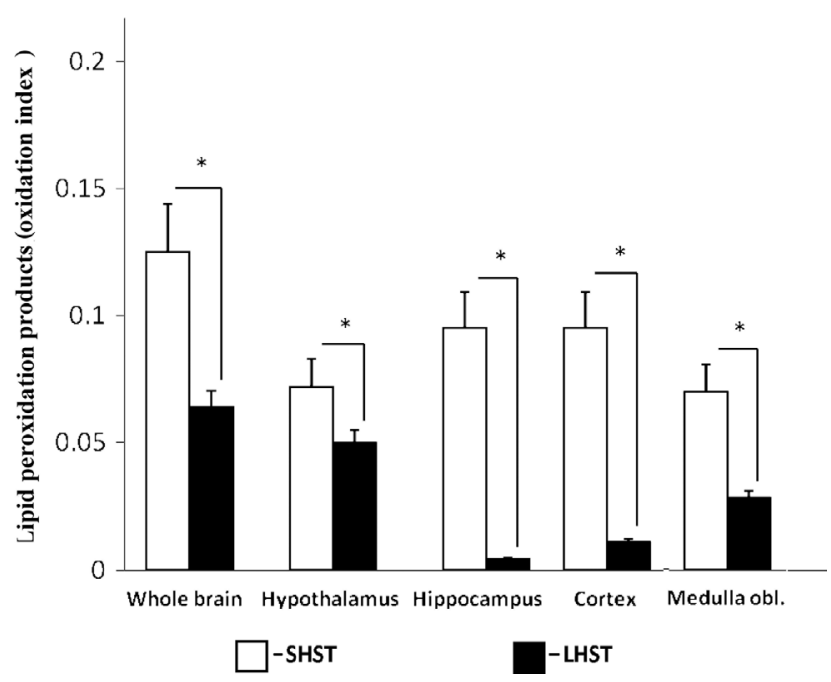

Figure 7. Lipid peroxidation products in rat brain. Both in the whole brain and all brain regions, content of heptane-soluble ketodiens and conjugated triens was higher for SHST rats (empty bars) than for LHST rats (black bars). Significant difference between groups, ${ }^{*} p<0.05,{ }^{* *} p<0.01$. 
the cortical level of heptane-soluble ketodiens ( $\mathrm{Rs}=-0.765$; $p=0.14)$. An interesting finding was a positive correlation between the whole brain content of carbonylated proteins and hepatic content of CYP $(0.689 ; p=0.023)$.

\section{Discussion}

It was suggested that the differences between SHST and LHST rats are explained by MAO activity in their brains and by central serotonergic regulation of liver microsomal oxidation. This hypothesis was based on the observations that intracerebral administration of serotoninergic neurotoxin increases mRNA levels of CYP1A1, CYP1A2, CYP2C11 and CYP3A1 (Rysz et al. 2015). It is therefore possible that brain serotoninergic system down regulates the expression of liver CYP (Rysz 2015). This suppressing effect of central 5-HT on liver CYP activity may also explain the differences in MAO-A activity between SHST and LHST rats. Our results showed that SHST rats with a higher level of microsomal oxidation had higher activity of brain MAO-A and MAO-B enzymes. Although MAO-B is not directly involved in serotonin metabolism, surprisingly high MAO-B expression is observed in serotoninergic neurons (Nagatsu 2004).

Cerebral MAO is involved in the regulation of certain behavioral activities due to its involvement in the metabolism of monoamine neurotransmitters. Changes in behavioral activity characteristic of the SHST group, including decreased horizontal activity and increased axiogenic defecation, were observed in animals exposed to restraint stress (Volchegorskii et al. 2004). In these stress-exposed animals, MAO activity was also increased. Consistently, in our experiments, SHST rats, characterized by higher MAO-A and MAO-B activities, showed lower locomotion and more frequent anxiogenic defecation than LHST rats. In addition, SHST rats demonstrated higher levels of lipid peroxidation products and carbonylated proteins, supporting the role of $\mathrm{MAO}$ in induction of oxidative stress.

Increased cerebral MAO activity has been associated with the development of anxiety and depression (Volchegorskii et al. 2004). Earlier, behavioral abnormalities similar to human melancholic depression were observed in rats after four restraint sessions one hour each with a 72 hour intervals between the restraint sessions (Volchegorskii et al. 2004). In that study, rats with increased MAO-B activity showed decreased locomotion and increased frequency of anxiogenic defecation. Using this model of anxiogenic stress, we observed that the development of anxiety and depression was associated with increased cerebral lipid peroxidation, which could be regulated with the MAO-B inhibitor, selegeline (Tseilikman et al. 2009). In the current study, MAO activity and free radical oxidation levels were not differ between dif- ferent brain regions. It is therefore useful to use the whole brains to assess these parameters.

It was reported that serotonin modulates the catalytic activity of CYP (LaBella et al. 2000). It may explain the differences in plasma serotonin levels between SHST and LHST rats. Our results showed that SHST rats simultaneously had lower levels of plasma serotonin and higher CYP content than LHST rats.

Up this date, no evidence on direct participation of CYP in metabolism of biogenic amines was available. It was recently reported that CYP isoform, CYP2D6, contributes to the transformation of 5-methoxytryptamine to serotonin (Haduch et al. 2013). It is therefore possible that CYP-dependent monooxygenation play a role in the regulation of biogenic amines, e.g., via the modulation of MAO activity. It was reported that the expression of genes coding for $\mathrm{MAO}-\mathrm{A}$ and $\mathrm{MAO}-\mathrm{B}$ is regulated by endogenous hormones, such as corticosteroids and sex hormones (Grunewald et al. 2012). However, liver CYP enzymes are metabolizing all steroid hormones (Niwa et al. 2015). This might be a possible mechanism for CYP-mediated regulation of central monoamines.

All differences between SHST and LHST rats observed long time after treatment. Our results suggest that the hexobarbital-induced sleep test might be an appropriate tool for the assessment of the status of central monoamine transmission. In addition to the overall intensity of microsomal oxidation, this test can indirectly characterize the integrative state of the neuro-endocrine factors regulating microsomal oxidation in vivo.

\section{Conclusion}

Data obtained in this study showed a correlation between MAO activity and microsomal oxidation phenotype. Rats with higher hepatic content of CYP had higher activities of MAO-A and MAO-B in the brain and higher plasma serotonin levels than rats with lower microsomal oxidation. These correlations may reflect the contribution of biogenic amines to regulation of CYP-dependent monooxygenases. Since brain serotonin system plays a central role in pathophysiology and treatment of depression, and since CYP reciprocally interacts with serotonin neurotransmission, liver microsomal oxidation may be a useful peripheral biomarker in the diagnostics of mood disorders and assessment of efficacy of antidepressant treatment. Further studies are required to elaborate this suggestion.

Acknowledgements. The study was supported by the Ministry of Education and Science of the RF as a part of base State Program (Project code 1696) and by grant 14-04-01381 from the Russian Foundation for Basic Research. 


\section{References}

Abell C. W., Kwan S. W. (2001): Molecular characterization of monoamine oxidase A and B. Prog. Nucleic Acid Mol. Biol. 65, 129-156 http://dx.doi.org/10.1016/S0079-6603(00)65004-3

Brunner H. G., Nelen M., Breakfield X. O. Ropers H. H., Van Oost B. A. (1993): Abnormal behavior associated with a point mutation in the structural gene for monoamine oxidase A. Science 262, 578-580 http://dx.doi.org/10.1126/science.8211186

Chen K., Shih J. C. (1998): Monoamine oxidase A and B: structure, function, and behavior. Adv. Pharmacol. 42, 292-296 http://dx.doi.org/10.1016/S1054-3589(08)60747-4

Cherala G., Shapiro B. H., D'mello A. P. (2007): Effect of perinatal low protein diets on the ontogeny of select hepatic cytochrome p450 enzymes and cytochrome p450 reductase in the rat. Drug Metab. Dispos. 35, 1057-1063 http://dx.doi.org/10.1124/dmd.106.013748

Duncan J., Johnson S., Ou X. M. (2012): Monoamine oxidases in major depressive disorder and alcoholism. Drug Discov. Ther. 6, 112-122 http://dx.doi.org/10.5582/ddt.2012.v6.3.112

Ekstedt B., Oreland L. (1976): Effect of lipid-depletion on the different forms of monoamine oxidase in rat liver mitochondria. Biochem. Pharmacol. 25, 119-124 http://dx.doi.org/10.1016/0006-2952(76)90277-X

Glover V., Sandler M., Owen F., Riley G. J. (1977): Dopamine is a monoamine oxidase B substrate in man. Nature $265,80-81$ http://dx.doi.org/10.1038/265080a0

Glowinski J., Iversen L. L. (1966): Regional studies of catecholamines in the rat brain. The diposition of $[3 \mathrm{H}]$ norepinephrine, $[3 \mathrm{H}]$ dopamine and $[3 \mathrm{H}] \mathrm{DOPA}$ in various regions of the brain. J. Neurochem. 13, 655-669 http://dx.doi.org/10.1111/j.1471-4159.1966.tb09873.x

Grunewald M., Johnson S., Lu D., Wang Z., Lomberk G., Albert P R., Stockmeier C.A., Meyer J. H., Urrutia R., Miczek K. A. et al. (2012): Mechanistic role for a novel glucocorticoid-KLF11 (TIEG2) protein pathway in stress-induced monoamine oxidase A expression. J. Biol. Chem. 287, 24195-206 http://dx.doi.org/10.1074/jbc.M112.373936

Furlong R. A., Ho L., Rubinsztein J. S., Walsh C., Paykel E. S. Rubinszlein D. C. (1999): Analysis of the monoamine oxidase A (MAO A) gene in bipolar affeclive disorder by association studies, mcta-analysis, and sequencing of the promoter. Am. J. Med. Genet. 88, 398-406

http://dx.doi.org/10.1002/(SICI)1096-8628(19990820) 88:4<398::AID-AJMG18>3.0.CO;2-Y

Haduch A., Bromek E., Sadakierska-Chudy A., Wójcikowski J., Daniel W. A. (2013): The catalytic competence of cytochrome P450 in the synthesis of serotonin from 5-methoxytryptamine in the brain: an in vitro study. Pharmacol. Res. 67, 53-59 http://dx.doi.org/10.1016/j.phrs.2012.10.009

LaBella F. S., Queen G. M., Brandes L. J. (2000): Interactive binding at cytochrome $\mathrm{P}-450$ of cell growth regulatory bioamines, steroid hormones, antihormones, and drugs. J. Cell Biochem. 76, 686-694 http://dx.doi.org/10.1002/(SICI)1097-4644(20000315)76:4<686:: AID-JCB16>3.0.CO;2-V
Levine R. L., Garland D., Oliver C. N., Amici A., Climent I., Lenz A. G., Ahn B. W., Shaltiel S., Stadtman E. R. (1990): Determination of carbonyl content in oxidatively modified proteins. Methods Enzymol. 186, 464-478 http://dx.doi.org/10.1016/0076-6879(90)86141-H

Kot M., Sadakierska-Chudy A., Haduch A., Rysz M., Bromek E., Gołembiowska K., Daniel W. A. (2015): The role of the dorsal noradrenergic pathway of the brain (locus coeruleus) in the regulation of liver cytochrome P450 activity. Eur. J. Pharmacol. $751,34-41$

http://dx.doi.org/10.1016/j.ejphar.2015.01.014

Miura H., Naoi M., Nakahara D., Ohta T., Nagatsu T. (1993): Changes in monoamine levels in mouse brain elicited by forcedstress, and the protective effect of a new monoamine inhibitor, RS-8359. J. Neural Trans. 94, 175-187 http://dx.doi.org/10.1007/BF01277023

Nagatsu T. (2004): Progress in monoamine oxidase (MAO) research in relation to genetic engineering. Neurotoxicology 25, 11-20 http://dx.doi.org/10.1016/S0161-813X(03)00085-8

Niwa T., Murayama N., Imagawa Y., Yamazaki H. (2015): Regioselective hydroxylation of steroid hormones by human cytochromes P450. Drug Metab. Rev. 47, 89-110 http://dx.doi.org / 10.3109/03602532.2015.1011658. Epub 2015 Feb 13 http://dx.doi.org/10.3109/03602532.2015.1011658

Omura T., Sato R. (1964): The carbon monoxide binding pigment of liver microsome. Evidence of haemoprotein nature. J. Biol. Chem. 239, 2370-2378

Papaliodis D., Boucher W., Kempuraj D., Theoharides T. C. (2008): The flavonoid luteolin inhibits niacin-induced flush. Br. J. Pharmacol. 153, 1382-1387 http://dx.doi.org/10.1038/sj.bjp.0707668

Pappas J. B., Franklin M. R. (1993): Hepatic clotrimazole concentrations and hepatic drug metabolizing enzyme activities in adult male Sprague-Dawley rats. Toxicology 80, 27-35 http://dx.doi.org/10.1016/0300-483X(93)90074-3

Rysz M., Bromek E., Haduch A., Sadakierska-Chudy A., Daniel W. A. (2015): Damage to the Brain Serotonergic System Increases the Expression of Liver Cytochrome P450. Drug Metab. Dispos. 43, 1345-1352 http://dx.doi.org/10.1124/dmd.115.064980

Satav J. G., Katyare S. S. (2004): Effect of streptozotocin-induced diabetes on oxidative energy metabolism in rat liver mitochondria-A comparative study of early and late effects. Indian J. Clin. Biochem. 19, 23-31 http://dx.doi.org/10.1007/BF02894253

Spina E., Trifirò G., Caraci F. (2012): Clinically significant drug interactions with newer antidepressants. CNS Drugs 26, $39-67$ http://dx.doi.org/10.2165/11594710-000000000-00000

Tipton K. F., Youdim M. B. H., Barwell C. J., Callingham B. A., Lyles G. A. (1992): Amine oxidase: function and dysfunction. J. Neural Trans. 41, 1-57

Tipton K. F., Davey G., Motherway M. (2006): Monoamine oxidase assays. Curr. Protoc. Toxicol. 4, 4.21

http://dx.doi.org/10.1002/0471141755.tx0421s30 http://dx.doi.org/10.1002/0471141755.tx0421s30

Tseilikman V. E., Sinitsky A. I., Poyarkov K. A., Vojdaev E. V. (2009): Effect of deprenyl on free radical oxidation in rat 
brain during immobilization stress. Bull. Exp. Biol. Med. 148, 856-858

http://dx.doi.org/10.1007/s10517-010-0835-1

Volchegorskii I. A., Nalimov A. G., Iarovinskiü B. G., Lifshits R. I. (1989): Comparison of various approaches to the determination of the products of lipid peroxidation in heptane-isopropanol extracts of blood. Vopr. Med. Khim. 35, 127-131 (in Russian)

Volchegorskii I. A., Tseilikman V. E., Smirnov D. S., Ship S. A., Borisenkov A. V. (2004): Decreases in glucocorticoid sensitiv- ity as a factor of stress-producing changes in the activity of monoamine oxidase, lipid peroxidation, and behavior in rats. Neurosci. Behav. Physiol. 34, 697-701

http://dx.doi.org/10.1023/B:NEAB.0000036010.90414.d9

Received: August 13, 2015

Final version accepted: September 20, 2015

First published online: December 22, 2015 